\title{
A DEEP, LARGE-AREA K-BAND SURVEY FOR HIGHLY REDSHIFTED H $\alpha$ EMISSION
}

\author{
P.P. VAN DER WERF \\ Leiden Observatory \\ P.O. Box 9513 \\ NL - 2300 RA Leiden \\ The Netherlands \\ (pvdwerf@strw.leidenuniv.nl)
}

\section{Introduction}

The most significant unknown in searches for high- $z$ starburst galaxies is the role and importance of absorption by dust. Emission line searches have mostly targeted the Ly $\alpha$ line, which, due to resonant scattering, will be strongly suppressed even if only small amounts of dust are present. Indeed, most of the high redshift starburst galaxies identified by Steidel et al. (1996) have no Ly $\alpha$ emission. The $U$-band and $V$-band dropout techniques developed by these authors and by Madau et al. (1996) are likewise sensitive to dust absorption, both because of absorption of the observed ultraviolet (UV) radiation and because UV-obscured galaxies will not be accounted for in these dropout studies.

Dust absorption is largely avoided by searching for $\mathrm{H} \alpha$ emission. We have carried out a deep, large-area near-IR survey for $\mathrm{H} \alpha$ emission at $z=2.1-2.4$, using the near-IR camera IRAC2B at the ESO/MPI $2.2 \mathrm{~m}$ telescope at La Silla, to image in total about $42 \square^{\prime}$ in $2 \%$ narrow-band filters to an r.m.s. noise of typically $10^{-16} \mathrm{erg} \mathrm{s}^{-1} \mathrm{~cm}^{-2}$, and in a broad-band $\mathrm{K}^{\prime}$ filter. We selected objects with significant excess narrow-band flux as emission-line candidates.

\section{Results and conclusions}

The limits of our survey are presented in Fig. 1, which shows that this survey for the first time probes all reasonable (evolved) $\mathrm{H} \alpha$ luminosity functions to some extent. Two serendipitous emission line objects were found. After correcting for the part of the luminosity function not sampled by our survey, a comoving star formation density of $0.12 M_{\odot} \mathrm{yr}^{-1}$ at $z=$ 


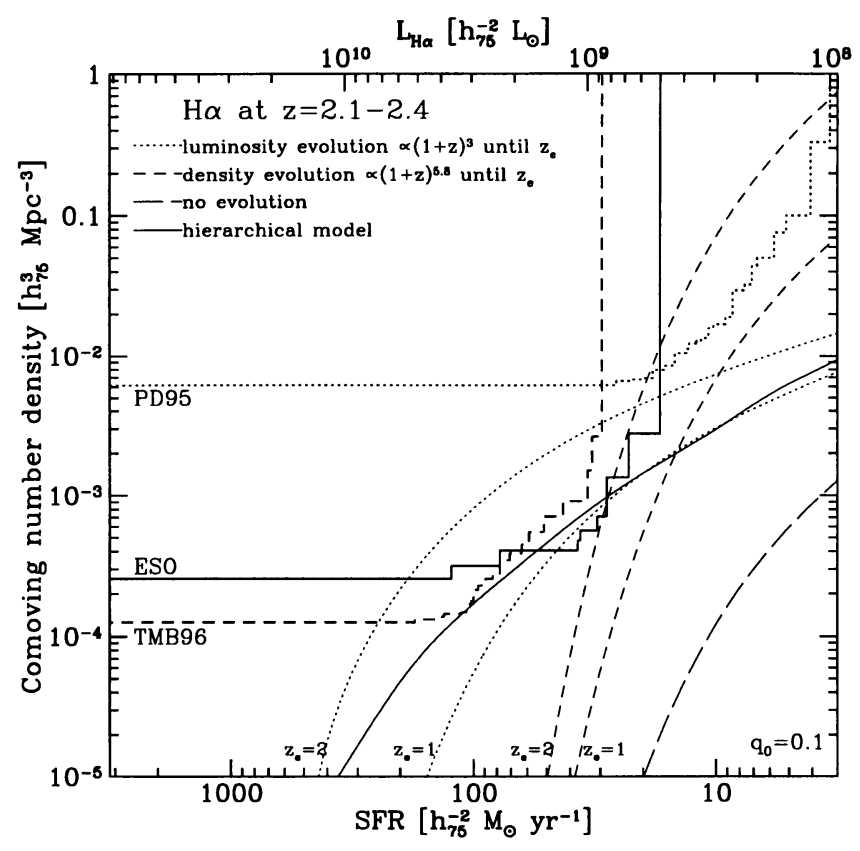

Figure 1. Limiting comoving volume densities at $90 \%$ confidence level implied by our survey (ESO) and the surveys by Thompson et al. (1996, TMB96) and Pahre \& Djorgovski (1995, PD95), for $H_{0}=75 \mathrm{~km} \mathrm{~s}^{-1} \mathrm{Mpc}^{-1}$ and $q_{0}=0.1$, as assumed throughout this paper. The curves indicate integrated Schechter-type luminosity functions based on the local $\mathrm{H} \alpha$ luminosity function (Gallego et al. 1995), labeled "no evolution", and evolved in luminosity or in density as indicated. The continuous curve results from a semi-analytical calculation of galaxy formation and evolution in a hierarchically clustering CDM universe (Baugh, priv. comm.).

2.25 results, where we have adopted the continuous curve in Fig. 1 as the luminosity function, a Salpeter Initial Mass Function, and the Kennicutt (1983) conversion factor from $\mathrm{H} \alpha$ luminosity to star formation rate. This survey shows the potential of future work with $8 \mathrm{~m}$ class telescopes, which will allow a determination of the $\mathrm{H} \alpha$ luminosity function at the peak of the AGN era, giving a reliable census of the star formation properties of the universe in this important epoch.

\section{References}

Gallego, J., Zamorano, J., Aragón-Salamanca, A., \& Rego, M. 1995, ApJ, 455, L1

Kennicutt, R.C. $1983, A p J, \mathbf{2 7 2}, 54$

Madau, P. 1996, MNRAS, 283, 1388

Pahre, M.A., \& Djorgovski, S.G. 1995, ApJ, 449, L1

Steidel, C.C., Giavalisco, M., Pettini, M. et al. 1996, ApJ, 462, L17

Thompson, D., Mannucci, F., \& Beckwith, S.V.W. 1996, AJ, 112, 1794 